\title{
Primary Particle Size Analysis of Gold Nanoparticles by Transmission Electron Microscopy and Optical Spectroscopy
}

\author{
A. Paez*, N. Ramani*, D. Kim**, C. Queenan**, A. Calabro**, J. Sankar*, D. Becker** \\ * Bergen County Technical Schools - Teterboro, 504 Route 46 West and Central Avenue, Teterboro, \\ NJ 07608 \\ ** Bergen County Academies, Nano-Structural Imaging Lab, 200 Hackensack Avenue, Hackensack, \\ NJ 07601
}

Gold nanoparticles (AuNPs) have become one of the most widely studied metallic nanomaterials for various applications, such as biosensors, catalysts and nonlinear optical materials [1] particularly because synthesis of AuNPs with good stability, controlled size and narrow size distribution can be easily achieved. In addition, AuNPs exhibit interesting optical properties known as surface plasmon resonance (SPR), changing colors from dark blue to ruby-red, depending on the size of the AuNPs.

Recently, the facile synthesis of AuNPs using citrate method originally developed by J. Turkevich et al. [2] has been modified to fit into nanotechnology classrooms of undergraduate and high school students [3]. Although the synthesis can be done within an hour with non-toxic chemicals $\left(\mathrm{HAuCl}_{4}\right.$ and sodium citrate) and the striking color change amazes many students involved in the lab, the understanding of AuNPs growth mechanism and the correlation of its color (or Visible absorbance) with their size has not been thoroughly investigated, but current research reports different mechanisms for the size-absorbance correlation [4], [5], [6]. Moreover, transmission electron microscopy studies of AuNPs synthesized using the citrate method in [3] reveals their polycrystallinity as shown in Figure 1, suggesting a complex growth mechanism of AuNPs which cannot be easily explained as a simple bottom-up approach.

In an attempt to understand the growth of AuNPs, aliquots of AuNPs were taken during the synthesis at different time intervals and quenched immediately with ice for analysis by transmission electron microscopy (TEM) and optical spectroscopy. The synthesis method is identical as reported in [3]. Primary particle size analysis was done using ImageJ software and compared to the peak wavelength of the visible absorbance spectra.

The series of visible absorbance spectra of AuNPs (Figure 3) shows a strong SPR peak at $524 \mathrm{~nm}$ emerging only after 5 minutes into the reaction. The broad absorption peaks of initial stages in the AuNPs formation (from 1 min to 3 mins) suggest the existence of aggregates, as confirmed in Figure 2. The primary particle sizes of these initial stages obtained from TEM studies are slightly bigger, but not as big as reported elsewhere. Future studies will be focused on the understanding of how this AuNPs synthesis method can be tailored to generate single crystalline AuNPs.

\section{References}

[1] M. Daniel et al., Chem. Rev. 104 (2004) 293-346.

[2] J. Turkevich et al., Discuss. Faraday Soc. 11 (1951) 11.

[3] A.D. McFarland et al., Journal of Chemical Education. 81(2004) 544A-544B.

[4] B. Pong et al., J. Phys. Chem. C 111 (2007) 6281-6287.

[5] J. Kimling et al., J. Phys. Chem. B 110 (2006) 15700-15707. 
[6] M.K. Chow et al., J. Coll. Interface Sci. 165 (1994) 97-109.

[7] The authors wish to thank the Carl D. Perkins Grant from New Jersey Department of Education for equipment purchase, training and maintenance used in this research.
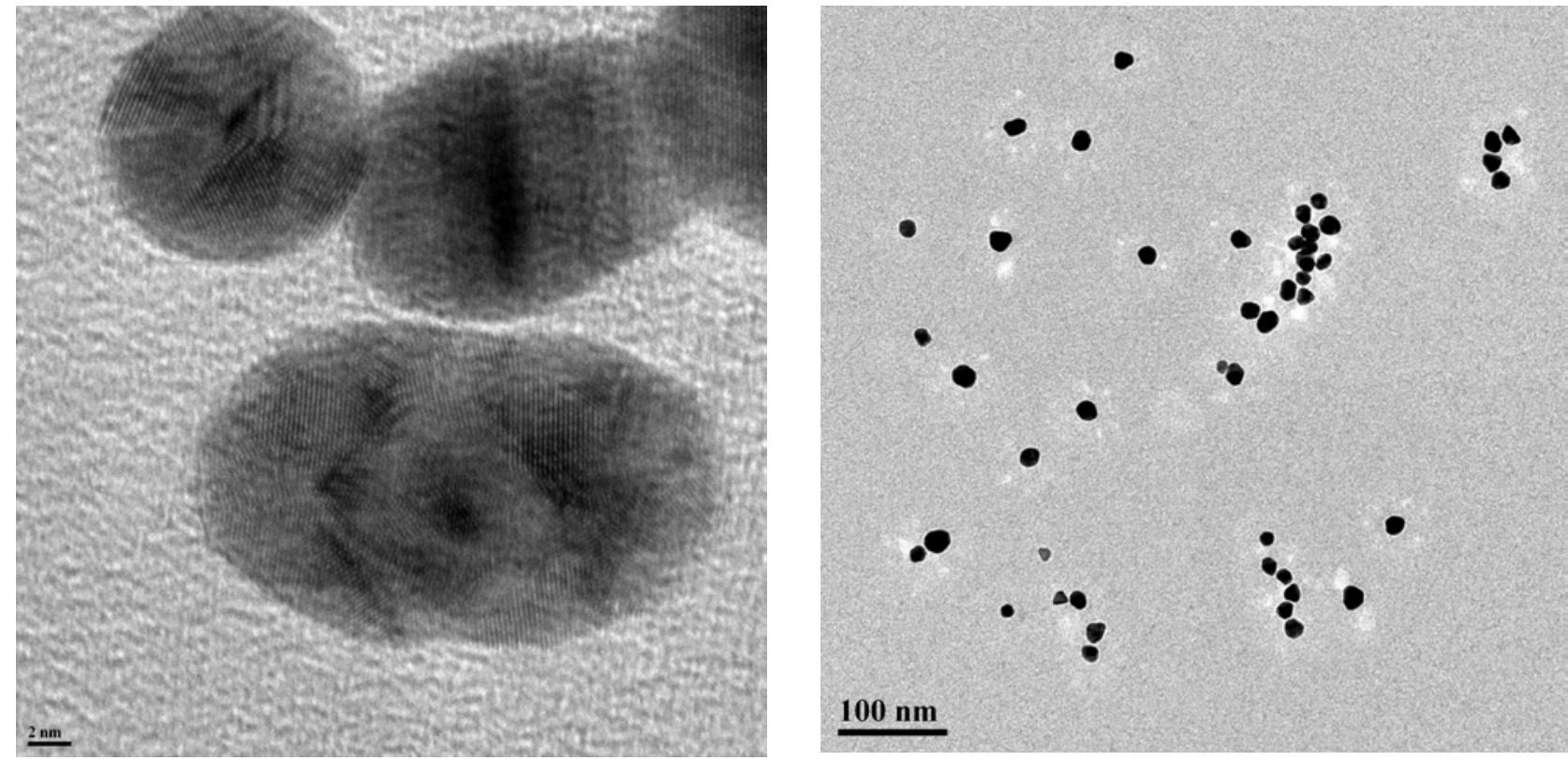

(LEFT) FIG. 1: High Resolution TEM image of polycrystalline gold nanoparticles synthesized by the citrate method after 20 minutes of reaction time. Scale bar, $2 \mathrm{~nm}$.

(RIGHT) FIG. 2: TEM image of gold nanoparticles after 1 minute of reaction time. Scale bar, 100 $\mathrm{nm}$
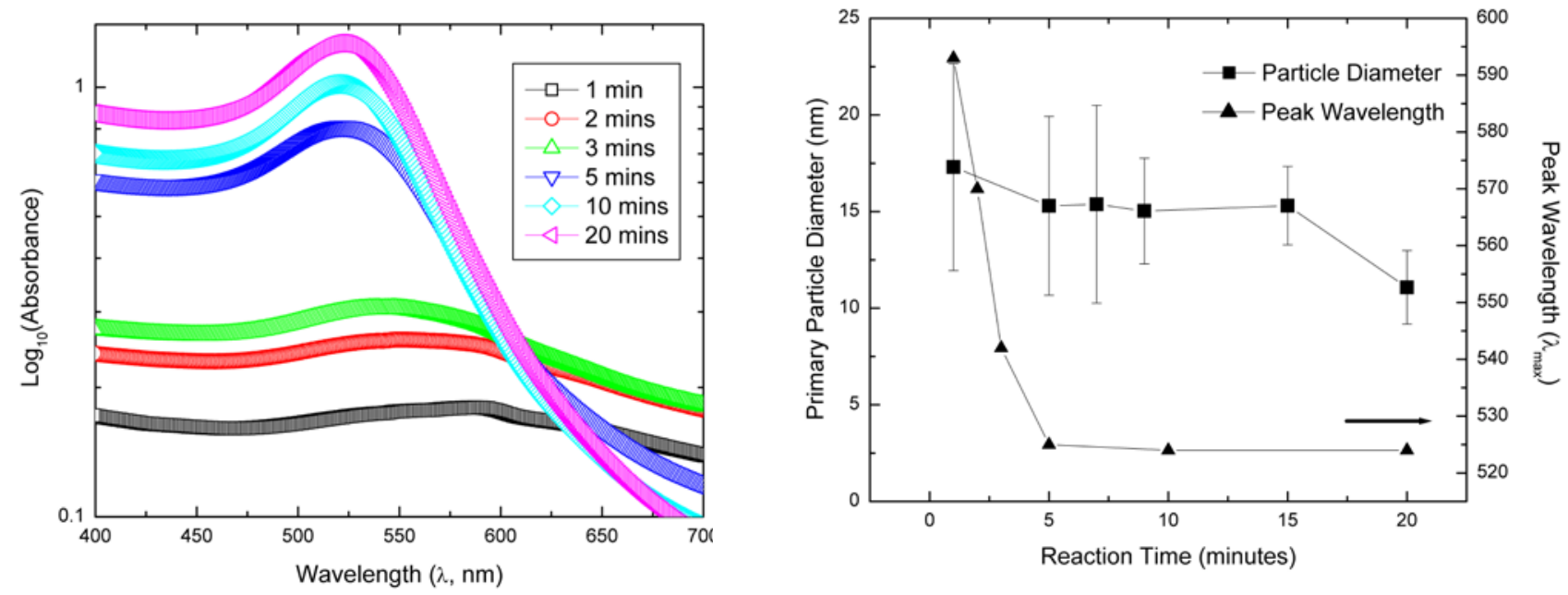

(LEFT) FIG. 3: Visible absorbance spectra of gold nanoparticle colloidal suspensions at different reaction time at $100{ }^{\circ} \mathrm{C}$.

(RIGHT) FIG. 4: Primary gold particle size change with different reaction times, and its correlation to the peak wavelength of visible absorbance spectra. Particle diameters are estimated with TEM viewgraphs using ImageJ software particle analysis function. 